\title{
The Effects of Internal and External Factors on the Mechanical Behavior of the Foam Copper
}

\author{
Longzhi Zhao ${ }^{1}$, Xiaolan Zhang ${ }^{1}, \mathrm{Na} \mathrm{Li}^{1}$, Mingjuan Zhao ${ }^{1}$, and Jian Zhang ${ }^{2}$ \\ ${ }^{1}$ School of Mechanical and Electronical Enginering, \\ East China Jiaotong University, Nanchang, \\ Jiangxi, P.R. China \\ zhaolongzhi@163.com, zhang_xiaolan85022@163.com \\ lina19841007@163.com, zhaomingjuan100@163.com \\ ${ }^{2}$ School of Mechanical Electronical Engineering, \\ Nanchang University, Nanchang 330013, China \\ zhj6115@126.com
}

\begin{abstract}
The study on the mechanical properties of foam copper has become increasingly important, for the potential market in the foam metal. The Kelvin model was supposed to be the suitable model to construct the ideal open-cell, continued and three-dimensional structure. The static tensile behavior on the foam copper was simulated by ANSYS and the effects of the porosity and strain rate on the foam copper were analyzed. The results show that there is a great influence of the porosity on the elastic modulus - the higher the porosity is, the smaller the elastic modulus, and though the larger the strain rate is, the smaller the elastic modulus, the effect on it is very small. The Kelvin model exhibit a characters of anisotropy, and the displacement and the stress of the node is different form its' location.
\end{abstract}

Keywords: ANSYS, foam copper, porosity, strain rate.

\section{Introduction}

The porous metal is a continued grid body which forms of strings or walls. The cavities is constructed by the three -dimensional polyhedron, and then called this kind of three-dimensional materials "foam materials". The composition of the foam solid is only by the cell edges (the cavities connected by the strings) called "open-cell", and called "closed-cell" for the walls of polyhedron is solid, and then the cavities is closed between its adjacent cavities[1]. With the rapid development of the computer technology, more and more heating of the CPU make obviously the CPU heat sink more important. For the heat sink materials is not satisfied the increasingly need of the market [2], zhang had invented a new heat sink material which called foam copper. So the mechanical properties of it are very significant and necessary.

Foam copper is a new functional material with three-dimensional pore structure, high porosity which can up to $60 \%$ 95\%, light specific gravity-only $10 \% \sim 50 \%$ of the 
same volume metal, high specific surface area can up to $10 \sim 40 \mathrm{~cm} 2, \mathrm{~cm} 3$ is widely used as heat exchangers and heat sinks. With three-dimensional copper foam pore structure, higher porosity, $60 \% \sim 95 \%$; proportion of light, with the volume of metal is only $10 \%$ to $50 \%$; the larger surface area, up to $10 \sim 40 \mathrm{~cm} 2, \mathrm{~cm} 3$, can be used for heat exchangers, and heat sinks[3]. In this paper, the static tensile behavior on the foam copper was simulated by ANSYS.

\section{The Establishment of the Model}

The Kelvin model is the ideal model that is used to simulate the mechanical properties of the lower relative density foam materials which can more truly reflect the geometric features of foam materials, which can fully fill the entire space, and can better meet the some properties. The unit cell of the Kelvin model is shown in Figure 1. What can be from the figure: it consists of 8 regular hexagons and 6 squares, contains 24 vertices and 36 pillars of equal length. Three plane-symmetry axises pass through the center of figure and four line-symmetry axises pass through the pillars of 6 squares [4]. The pillars of foam materials are conceived as same cross-section beam [5]. Kelvin multi-cell model is shown in Figure 2.Circle cross-section beam was established in this paper, and the load is imposed parallel to the direction of the extension of the cell. The $\mathrm{Y}$ axis is opposed to the direction, and the origin is showed at the bottom of the multi-cell model.

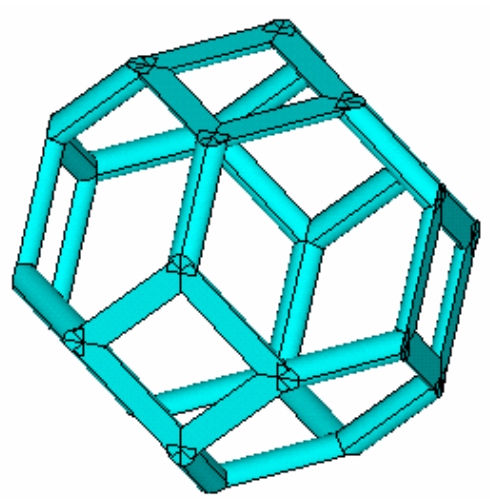

Fig. 1. The Kelvin model

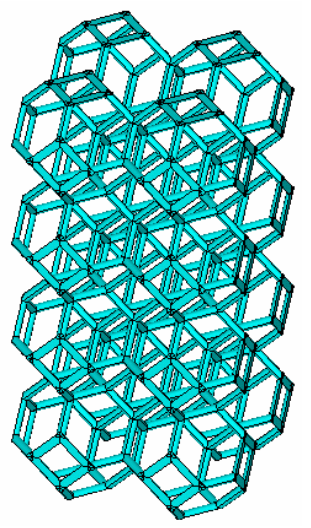

Fig. 2. The multi-cell model

\section{Discussion}

\subsection{The Effects of the Volume Fraction}

The volume fraction of the foam copper is changed by the porosity in this paper, and this means that the volume fraction is equal to the porosity in this paper. The porosity is one of the main characteristic parameters of the porous materials, which is referred to the volume of the solid compared to all of the volume. The porosity of foam is arranged 
between $85 \%$ and $95 \%$ in this paper. The mechanical properties and physical properties in the elastic area were studied in this paper. The volume fraction of the Kelvin model is shown in Eq.1.

$$
P=4 \sqrt{3} \pi\left(\frac{R}{a}\right)^{2}-(\sqrt{6}+\sqrt{2})\left(\frac{R}{a}\right)^{3}
$$

Where $\mathrm{P}$ is porosity, $\mathrm{R}$ is the radius of cross section, a length of the pillar. The materials parameters are shown in Table 1.

Table 1. The materials parameters

\begin{tabular}{cccccc}
\hline Porosity & $\begin{array}{c}\text { Section } \\
\text { radius } \\
\text { /mm }\end{array}$ & $\begin{array}{c}\text { elasticity } \\
\text { modulus } \\
\text { /Gpa }\end{array}$ & $\begin{array}{c}\text { Poisson's } \\
\text { ratio }\end{array}$ & $\begin{array}{c}\text { shear } \\
\text { modulus } \\
\text { /Gpa }\end{array}$ & $\begin{array}{c}\text { yield strength } \\
\text { /Mpa }\end{array}$ \\
\hline $85 \%$ & 0.0550 & 16.50 & 0.343 & 6.00 & 1.60 \\
$87 \%$ & 0.0515 & 14.00 & 0.343 & 5.50 & 1.40 \\
$89 \%$ & 0.0480 & 12.00 & 0.343 & 5.00 & 1.22 \\
$91 \%$ & 0.0440 & 9.90 & 0.343 & 4.14 & 1.00 \\
$93 \%$ & 0.0375 & 7.70 & 0.343 & 3.20 & 0.78 \\
$95 \%$ & 0.0315 & 5.50 & 0.343 & 2.30 & 0.56 \\
\hline
\end{tabular}

The time-displacement curves of three nodes are selected to analyze the mechanical properties of the foam copper based on the Kelvin model in this paper. The node 1156 located on the top surface, the node 3027 located on the middle of the model, and the node 11149 is closed to the bottom surface. The Y-axis time-displacement curves of three nodes are shown in the Figure 3. The results show that the increment in displacement is very small before the time of 0.1 second, the increment become large and increase linearly with the extend of the time. After stretching the model, the displacement of the node 1156 is larger than the node 3027, which is larger than the node 11149. These conclude that the more closed of the location to the force, the larger of the displacement is. This can owed to the time and space for the transmission of the force.

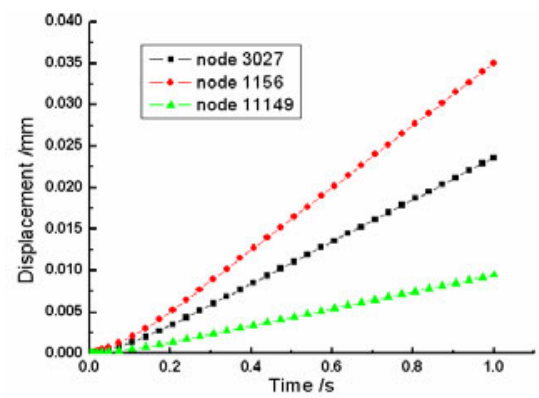

Fig. 3. Y-axis displacement

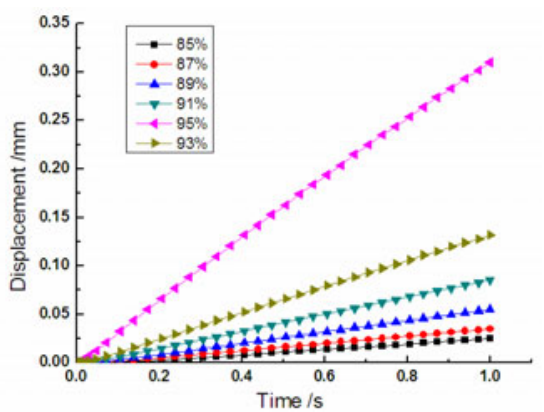

Fig. 4. Time-displacement curves under different porosity 
Figure 4 shows the time-displacement curves of the node 1156 under different porosity. It shows that in the elastic area, the greater the porosity, the smaller the displacement, which indirectly indicates that the greater the porosity, the elastic modulus. This manly result from that the smaller the porosity, the bigger the volume of entity, and the bigger load area, then the smaller the load of average area.

The time-displacement curves of X, Y, Z axes are shown in Figure 5. What can be from the figure is that the time-displacement curves of three axes trend differently. $Y, Z$ axes exhibit increasing trend, while $\mathrm{X}$ axis exhibit opposite. The increment of the $\mathrm{Z}$ axis is small than $\mathrm{Y}$ axis, for the $\mathrm{Y}$ axis is the direction of the force. So the Kelvin model has anisotropy character. This is accord with the LU's statement.

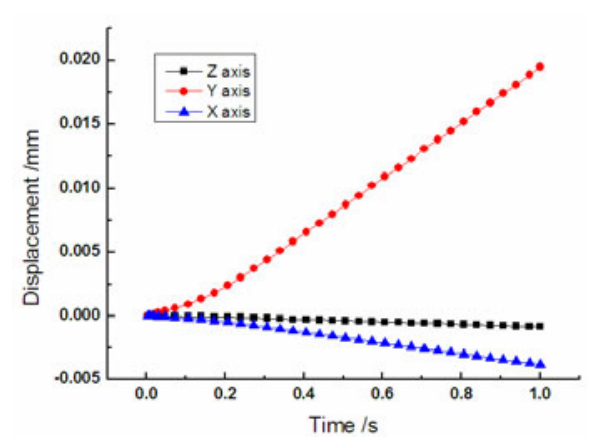

Fig. 5. Time-displacement curves of $\mathrm{X}, \mathrm{Y}$, Z-axes of the node 1156

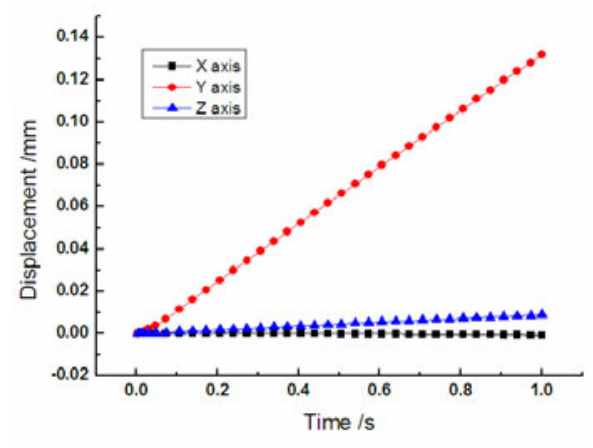

Fig. 6. Time-displacement curves of $\mathrm{X}, \mathrm{Y}$, Z-axes of the node 3027

\subsection{The Effects of the Strain Rate}

The porosity of the foam copper is set as $85 \%$, and the model is the same to above. The effect of the strain rate on the elastic modulus is shown in Figure 7. It can be seen from the figure that the elastic modulus doesn't exhibit linearly increase with the strain rate. The larger the strain rate, the smaller increment of the elastic modulus. The elastic modulus decrease, but it decreases small. Then can conclude that the effect of the strain rate on the elastic modulus is very small. It may be appearing a situation, the elastic modulus may be stable when the strain rate up to a limit. This is equal to the elastic modulus has a limit. This because that the strain rate would not neither change the internal structure and properties like the porosity and the aperture nor alter the external circumstance in order to change the internal properties like the temperature.

The Y-axis strain rate-displacement curves of three nodes located differently are shown in Figure 8. It shows apparently that the curves of the node 1156, 3027, and 11149 don't intersect, and different greatly. Especially in the situation of the small effect of the strain rate on the elastic modulus, the increment of the node's displacement depend on the location very largely. This indicates that the node distance from the force affect directly the stress and the displacement. This is because that though in static model, the more far to the load, the longer the time of the transmission, for the time and the space is necessary for the transmission of the load. And in the process of 
transmission, it is inevitable for the loss of the force. Then the displacement and stress is small with distant location. The Equivalent stress nephograms of the different location of the node are shown in Figure 9. The stress is increased gradual from blue to red. The same conclusion can be from the figure. What can be seen from the figure is that the stress is the biggest in the intersection. Although the angles between the pillars are not right angle, it is not beneficial to transfer the force especially in the intersection which is easy to concentrate the stress.

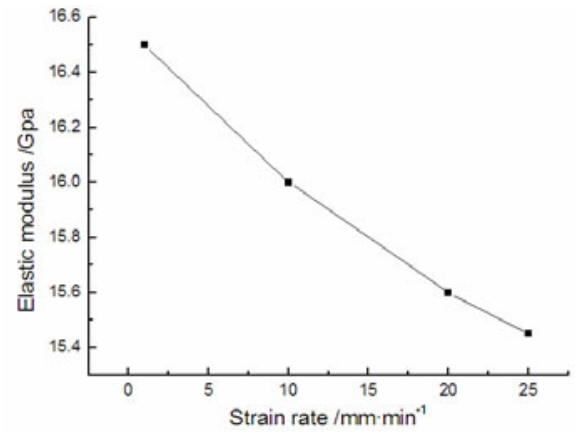

Fig. 7. Elastic modulus-strain rate curve

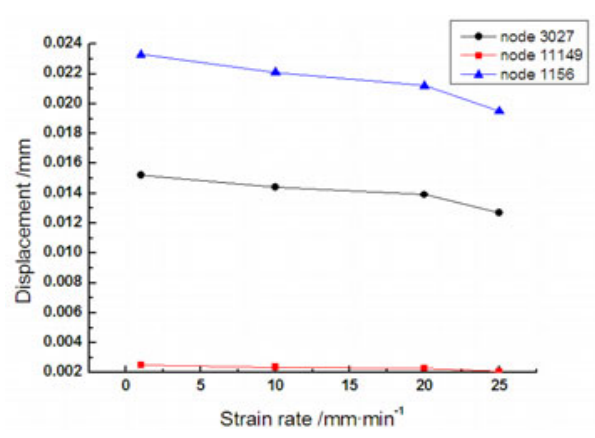

Fig. 8. Displacement-strain rate curves of different location

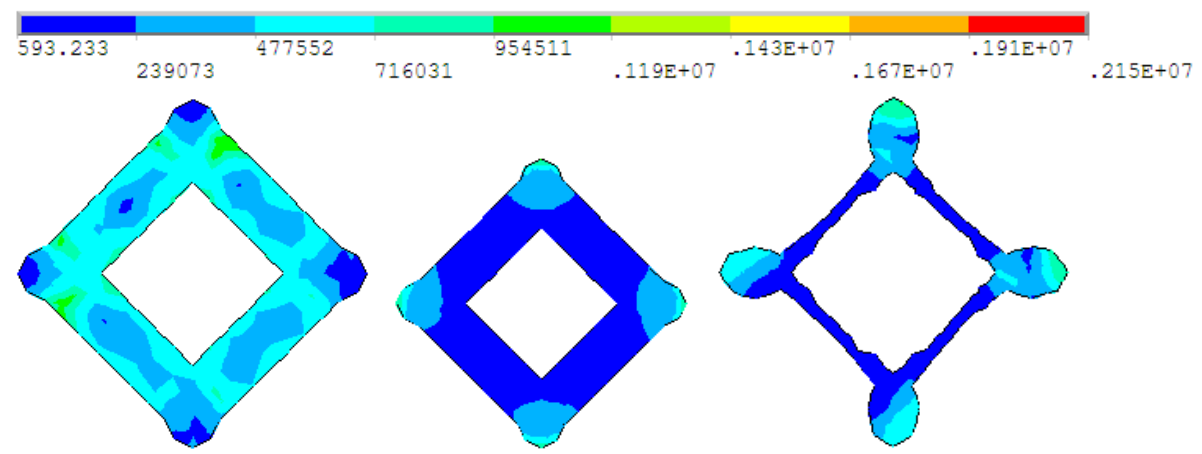

Fig. 9. Equivalent stress nephograms of the different location

\section{Conclusion}

(1) The elastic modulus varies largely under different porosity. The elastic is high increase with the decreases of the porosity.

(2) The elastic modulus decrease with the strain rate increasing, but the range is very small.

(3) The stress and displacement largely depend on the location from the load, and the stress and displacement is larger with closed distance.

(4) The Kelvin model exhibits anisotropy character. 


\section{References}

1. Zhang, J.Y., Zhang, P., Gan, Q.L., Xiao, Y.X.: The Properties of Representative Units of Porous Materials. J. Engineering Mechanics 21(2), 124-129 (2004)

2. Hao, Y.G., Liu, L.: Thermal design of electronic equipment. J. Ordnance Industry Automation 29(2), 49-54 (2010)

3. Cao, G.Y., Wang, F., Wang, L.C.: The Mechanical Property and Progress of Metal Foams. J. Research Studies on Foundry Equipment 2, 51-54 (2008)

4. Shi, S.L., Lu, Z.X.: Finite Element Analysis for The Elastic Modulus of Open-cell Foams Based on a Tetrakaidecahedron Model. J. Journal of Mechanical Strength. Henan. 28, 108-112 (2006)

5. Xie, L.S., Chen, M.H., Tong, G.Q., Gao, L.: Anisotropic Elastic Properties of Foam Metal with Open Cells. J. Materials for Mechanical Engineering 27, 7-16 (2003)

6. Lu, Z.X., Huang, J.X., Chen, X.: Elastic Properties of Anisotropic Kelvin Model for Open-cell Oams. J. Acta Aeronautica Et Astronautica Sinaca 6, 1017-1022 (2009) 